\title{
Polymer Sample Preparation for Electron Microscopy
}

\author{
Amanda M. Schrand* \\ *Graduate Department of Materials Engineering, The University of Dayton, 300 College Park, \\ Dayton, OH, 45469
}

Microscopy generally involves either the examination of a surface in reflection or the transmission of radiation through a thin sample [1]. The quality of the information obtained with microscopy is dependent on resolution and contrast [2]. Therefore, complementary techniques such as light and electron microscopy can impart valuable scientific insights. Light microscopy can initially save time and effort by providing a lower magnification overview to locate specific areas, check orientation, or discard uninteresting or poorly processed material [3]. However, features smaller than the wavelength of light are not resolvable with light microscopy. In order to visualize details that are orders of magnitude smaller, the scanning electron microscope (SEM) and transmission electron microscope (TEM) are utilized (Table 1).

Unlike light microscopes, electron microscopes require high vacuum levels in order for electrons to reach the sample without being scattered by atmospheric particles. Once the electrons reach the sample, they must interact in such a way to produce useful information. This information is generally in the form of a grayscale image. Image contrast in electron microscopes is produced through electron scattering by the atomic nuclei of the sample. In organic polymers, which consist exclusively of light atoms such as carbon, hydrogen, oxygen, and nitrogen, the scattering is weak and therefore produces poor contrast [3]. To meet these conditions needed for successful electron microscopy, polymers require special sample preparation (Table 2).

Ideally, samples for electron microscopy should be non-volatile, free of contaminants, able to withstand electron beam irradiation, produce atomic-based contrast, and act as conductors or be thin enough for electron transmission. Most polymers do not naturally possess these characteristics, which creates obstacles to having the best imaging conditions. These obstacles can be combated and overcome by proper cleaning and drying, etching and staining of low contrast samples, lightly coating to prevent charging and protect from beam damage, making a pathway to ground with conductive paint or tape, and sectioning with an ultramicrotome for ultrathin sections [1-7].

A successful SEM sample has a clean, supported, dry, conductive surface with a path to ground that can withstand electron beam irradiation and produce atomic contrast. A successful TEM sample is very thin ( $<100$ nanometers) in addition to all of the above characteristics. Although the sample is typically altered in the sample preparation process, the end goal is to retain its structural integrity and to not compromise the electron microscope [4]. 
References

[1] A. Vaughan, Polymers and Microscopy, RMC/Boeckeler 7th Annual Materials Microtomy Short Course, Tucson, 2002.

[2] D. T. Grubb and L. C. Sawyer, Polymer Microscopy, 2nd ed., Chapman and Hall, London, 1996.

[3] N. Reid, Ultramicrotomy, In: A. Glauert (editor), Practical Methods in Electron

Microscopy, Vol 3, North Holland Publishing Col, Amsterdam, 1974.

[4] J. Goldstein, D. Newbury, D. Joy, C. Lyman, P. Echlin, E. Lifshin, L. Sawyer, and J. Michael, Scanning Electron Microscopy and X-ray Microanalysis, 3rd ed., Kluwer Academic/Plenum

Publishers, New York, 2003.

[5] T. Malis, TEM Specimen Preparation “At the Cutting Edge”, Leica 2002 Ultramicrotomy of Industrial Materials, 1996.

[6] A. W. Robards and A. J. Wilson, Procedures in Electron Microscopy, John Wiley \& Sons, Chichester, 1993.

[7] C. Schooley, Materials Microtomy, RMC/Boeckeler 7th Annual Materials Microtomy Short Course, Tucson, 2002.

TABLE 1. Comparison of Optical and Electron Microscopes [2]

\begin{tabular}{lllll}
\hline Ranges & Stereo binocular & Compound light & SEM & TEM \\
\hline Useful magnifications & $5-100 \mathrm{x}$ & $30-1500 \mathrm{x}$ & $20-60 \mathrm{kx}$ & $3-250 \mathrm{kx}$ \\
Typical resolutions & 10 microns & 1 micron & 10 nanometers & 1 nanometer \\
$\begin{array}{l}\text { Best resolution } \\
\text { Smallest observable }\end{array}$ & 2 microns & 0.2 microns & 4 nanometers & 0.2 nanometers \\
polymer structures & Macroscopic & Spherulitic & Lamellar & Crystal lattice \\
Field of view & Very large & Large & Large & Small \\
& 5mm, 50x & 2mm, 50x & 20 microns, 5kx & 2 microns, 50kx \\
Imaging system & Light optical & Light optical & Non-optical & Electron optical \\
& Glass lenses & Glass lenses & Raster & Magnetic lenses \\
Sample environment & Ambient & Ambient & High vacuum & High vacuum \\
Radiation damage & None & None & Little & Severe \\
Chemical analysis & Not usually & Not usually & Yes, x-ray & Yes, x-ray
\end{tabular}

TABLE 2. Summary of Sample Preparation Techniques for Electron Microscopy [1-7]

\begin{tabular}{ll}
\hline Preparation & Specific Methodology \\
\hline Cleaning & Alconox detergent, plasma (etch), $\mathrm{CO}_{2}$ snow, acetone and alcohols, air duster \\
Fracturing & Controlled with machine or cool sample to temperature $<\mathrm{Tg}$, then break \\
Polishing & Silicon carbide, aluminum or chromium oxide, diamond paste, colloidal silica \\
Etching & Benzene, toluene, xylene, acetone, acids (nitric, chromic, sulfuric, orthophosphoric) \\
Staining & Osmium or ruthenium tetroxide, phosphotungstic acid, hydrazine, silver sulfide \\
Embedding & Epon 812 (epoxy), Spurr (epoxy), Araldite 6005 (epoxy), LR White (acrylic) \\
Sectioning & Ultrathin samples to 10's of nanometers, cut with diamond knife, can be cryo \\
Supporting & Aluminum or carbon stubs, carbon paint, copper tape, silver epoxy, copper grids \\
Coating & Carbon, gold, gold-palladium, tungsten, chromium, platinum, titanium \\
Storing & Vacuum dessicator or dry, dust-free, airtight container, constant temperature, label
\end{tabular}

